$\mathrm{DE}$

M E D I C I N A

T R O P I C A L

$\mathrm{DE}$

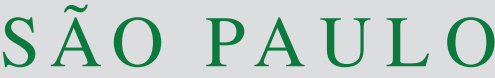

JOURNAL OF THE SÃO PAULO INSTITUTE OF TROPICAL MEDICINE

\section{Mycobacterium marinum infection simulating chromomycosis: a case report}

Guilherme Holanda Bezerra $^{\left({ }^{1}\right.}$, Monica Larissa Padilha Honório ${ }^{\circledR 1}$, Vivianne Lira da Camara Costa ${ }^{(1)}$, Hareton Teixeira Vechi ${ }^{\left({ }^{1}\right.}$, Manoella do Monte Alves $^{(1}$, Maria Helena Marques Fonseca de Britto ${ }^{(1)}$, Keyla Borges Ferreira Rocha ${ }^{(1}$, Luciana Distásio de Carvalho ${ }^{\circledR} 2$

\section{ABSTRACT}

Skins infections caused by Mycobacterium marinum occur only rarely. We report one case of chronic and extensive $M$. marinum cutaneous infection simulating chromoblastomycosis and review the pertinent literature. A 52-year-old farmer reported a 32-year chronic skin problem on his right lower limb, resulting from contact with cacti. It consisted of skin lesion presenting with dyschromic atrophic center plate and verrucous borders with hematic crusts, extending from the knee anteriorly to the inferior third of the right leg. Mycobacterium marinum infection was detected by histopathological examination of a skin fragment, culture for mycobacteria and genetic mapping of the culture material. The patient was successfully treated with Ethambutol, Rifampicin and Trimethoprim-Sulfamethoxazole. The clinical and histopathological findings of M. marinum infection is nonspecific showing clinical polymorphism and bacilli are rarely evident on histopathological examination. Given these difficulties, it is essential to perform tissue culture in a suspicious case and it is important keep this infection in mind in patients with long-lasting indolent verrucous lesions and a history of exposure to sea water, freshwater, aquaria or fish.

KEYWORDS: Atypical mycobacteria. Non-tuberculous mycobacteria. Mycobacterium marinum.

\section{INTRODUCTION}

Cutaneous infections caused by Mycobacterium marinum (Mma), a member of the nontuberculous mycobacteria (NTM) group, is a rare disease. M. marinum virtually always causes cutaneous disease through direct inoculation, typically following traumatic injuries caused by a contaminated environmental source $e^{1,2}$.

The manifestations include granulomatous lesions, predominantly acral in distribution, that affect patients regardless of their immune status. Clinical and histopathological findings are non-specific, consisting of papules, nodules or erythematous plaquesand the presence of tuberculous granulomas with little evidence of bacilli on examination. Due to diagnostic difficulties, going from the infrequent distribution and the plurality of clinical presentations, M. marinum can cause erythematous to plate-shaped, papules, nodules, single or multiple ulcerations and even sporotrichosis-like presentations, making the differential diagnosis with other granulomatous lesions essential ${ }^{3}$.

The infection in humans, due to contact with contaminated water or fish is comparatively rare with an incidence of 0.04 to 0.27 cases per 
100,000 inhabitants per year ${ }^{4}$. First described as a "swimming-pool granuloma", M. marinum skin infection is nowadays acquired in the maintenance of aquariums and, therefore, is called "fish tank granuloma" or aquarium granuloma ${ }^{5}$.

Mycobacterium marinum is an acid-fast, aerobic, not mobile, non-sporulating, photochromogen bacterium with a thick cell wall made up of wax (40-70\%) and lipids (25-30\%). Mycobacterium marinum growth is usually slow, takingtwo to six weeks on Lowenstein-Jensen medium at $25-33{ }^{\circ} \mathrm{C}$. By using light source in culture, the color changes from white to yellow because of the accumulation of $\beta$-carotene crystals. It has a worldwide diffusion, mainly in temperate and hot-humid climate, living in aquatic environments, especially in salt water and aquariums or pools ${ }^{4,6}$. This mycobacterium can infect cold-blooded animals such as turtles, amphibians and snakes, causing chronic systemic infection in fish ${ }^{4,7}$. Dead fish can serve, as well, as reservoirs ${ }^{4,8}$.

The diagnosis can be made by histological examination and identification of M. marinum in a culture of a fragment of a lesion fragment sown in Löwenstein-Jensen medium. The organisms isolated from the lesion, as well as from the patient's colonies are smooth, shiny and creamy colored, turning yellow under exposure to light (photochromogenic). Faster identification may be achieved by the polymerase chain reaction ${ }^{9}$. Dermatoscopic characteristics of a microbiologically-proven M. marinum skin infection can be used in the future to establish very early diagnosis of this infection, reducing the diagnostic delay ${ }^{10}$.

Here we report one case of atypical M. marinum cutaneous infection from an injury caused by contact with a cactus and review the pertinent literature.

\section{CASE REPORT}

A 52-year-old black male farmer from a rural area in Northeastern Brazil was referred to our outpatient infectious diseases clinic because of skin plaques after a trauma caused by a cactus thorn on his right leg 32 years before. He was otherwise healthy and mentioned bathing in ponds, fishing activities and handling fish for his own consumption. The plaque had a dyschromic atrophic center and erythematousviolaceous infiltrated verrucous borders with hematic crusts extending anteriorly from the upper border of the right knee to the lower third of the right leg (Figures 1A and 1B). He denied systemic symptoms, renal and hepatic functions, blood countand lipid profiles were within normal limits. A skin ellipse biopsy of the right leg was performed and showed a chronic inflammatory process in the superficial and deep dermis associated with pseudo-epitheliomatous hyperplasia (Figures 2A and 2B). Direct search for fungus and mycobacteria with Periodic acid-Schhiff (PAS) and Fite-Faraco, respectively, were negative (Figure 2C). A fungal culture was also negative. A mycobacteria culture in Ogawa Kudoh medium was positive (Figure 3) and M. marinum was identified through a Polymerase Chain Reaction (PCR) followed by a Restriction Enzyme Analysis (REA).

The patient received a combined treatment for six months composed of ethambutol $400 \mathrm{mg}$ three times a day for three months, rifampicin $300 \mathrm{mg}$ twice daily for six months and trimethoprim-sulfamethoxazole $(160 \mathrm{mg}+800 \mathrm{mg})$ twice daily for six months; topical treatment was not performed. The verrucous lesions disappeared (Figure 1C), leaving a dystrophic atrophic plaque in the affected region, with no relapse to date.

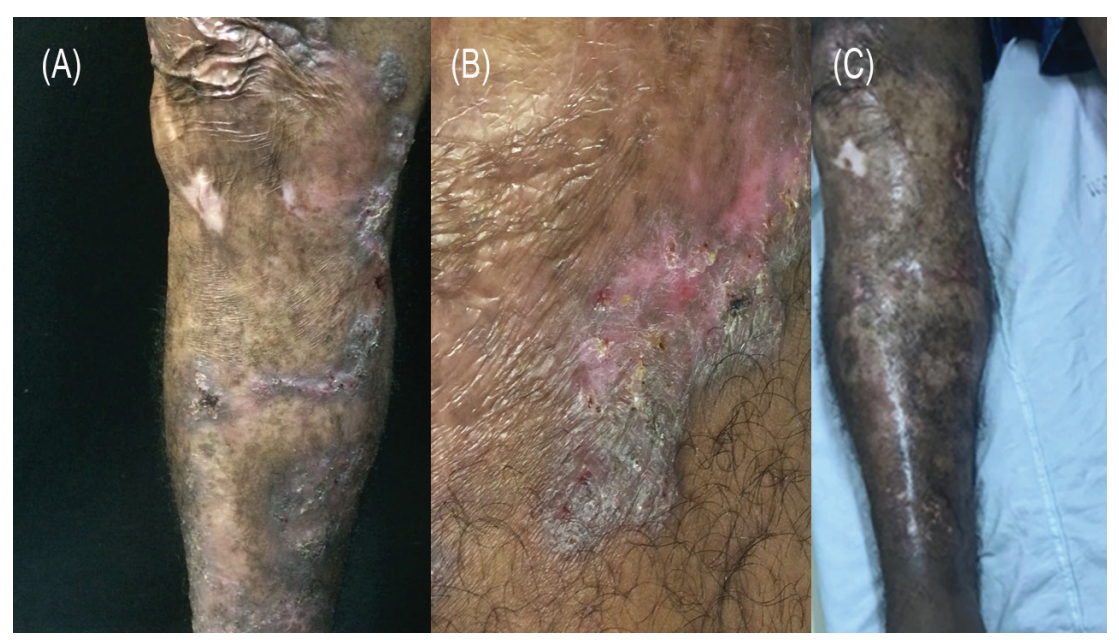

Figure 1 - Erythematous-violaceous infiltrated verrucous borders lesion with hematic crusts extending anteriorly from the upper border of the right knee to the lower third of the right leg $(A)$ and a plaque with a dyschromic atrophic center (B). Regression of cutaneous lesions six months after the beginning of treatment with an atrophic plaque in the entire region, previously affected, on right lower limb (C). 


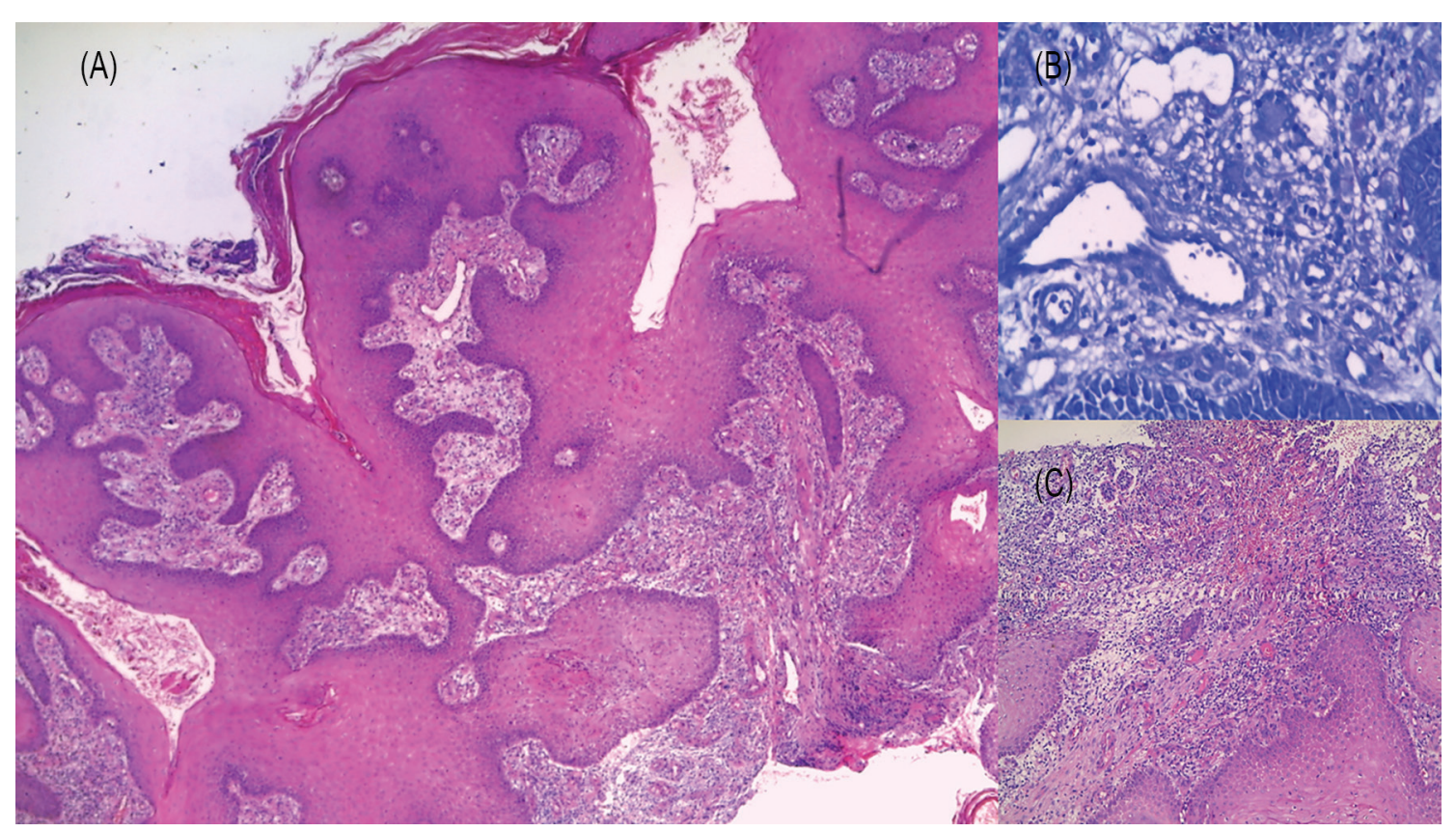

Figure 2 - Chronic inflammatory process in the superficial and deep dermis associated with pseudo-epitheliomatous hyperplasia with negative Periodic acid-Schhiff (PAS) (A, B). Acid-fast Bacilli (AFB) by Fite-Faraco negative to mycobacteria (C).

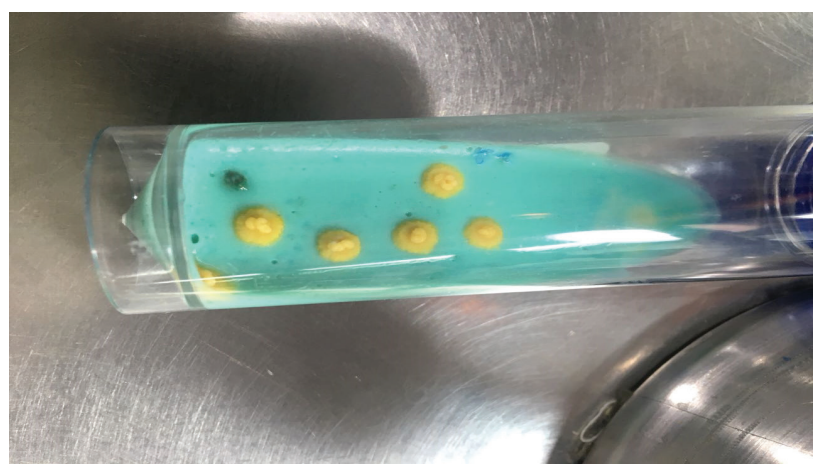

Figure 3 - Biopsy culture in Ogawa Kudoh medium culture showing colonies of M. marinum.

\section{DISCUSSION}

The genus Mycobacterium comprises more than 177 species including pathogenic, opportunistic pathogens and non-pathogenic environmental species, most of which have been incriminated in skin and soft tissues infections. Mycobacteria are free-living, acid-fast, robust organisms that can survive and even thrive in widely diverse environments such as soil and tap water to animals and humans consumption. Of interest to this study, M. marinum was first described in 1926 by Joseph D. Aronson. The bacterium was isolated from fish presenting with mycobacteriosis infection and exhibiting similarities with tuberculosis in humans. Later, it was shown that M. marinum could also infect humans and cause skin lesions at the extremities of the limbs ${ }^{11}$. Scientific interest in M. marinum is mainly due to its genetic relatedness to M. tuberculosis, and it is a model for tuberculosis pathogenesis ${ }^{12}$.

The retrospective study in Denmark performed from January $1^{\text {st }}, 2004$ to May $31^{\text {st }}, 2017$ identified 55 patients with culture-positive $M$. marinum infections. Men comprised $71.7 \%$ of patients with a median age of 49 years. Almost all patients had a history of aquatic exposure, predominately the maintenance of fish tank

Mycobacterium marinum infection usually occurs in immunocompetent patients with minor trauma followed by contact with an infected animal or handling of contaminated aquariums or water ${ }^{3}$. In immunocompetent patients lesions are usually solitary, a red-to-violaceous plaque or nodule with an overlying crust or verrucous surface that may ulcerate. Inflammatory nodules or abscesses can develop in severely immunosuppressed patient, usually in a type of distribution of sporotrichosis 9 . Our patient had been injured via puncture by a cactus spine to the knee. M. marinum can also cause an opportunistic infection, primarily in immune deficient patients, such as those with the human immunodeficiency virus (HIV)/acquired immunodeficiency syndrome (AIDS).

Mycobacterium marinum infection has polymorphic clinical presentations. Initially, a solitary erythematous papule or nodule will appear at the site of inoculation, often on an extremity, particularly the hand. This can progress to a verrucous violaceus plaque and/or ulcerate lesion producing a serosanguineous discharge, and in $29 \%$ of the cases, bacteria can invade deeper tissues, such as tendon sheaths, bursae, bones and joints. There a are four clinical 
categories according to the severity of infections: localized superficial cutaneous lesions (type I), nodular lymphangitis sometimes with nodules, abscesses and granulomas (type II), deeper infections with or without skin involvement (type III) and M. marinum disseminated infection with lung involvement and other systemic manifestations (type IV). Bacteremia is exceedingly rare but may occur in severely immunocompromised patients. Other sites of M. marinum infection have also been reported. Severe intranasal lesions resulting from $M$. marinum infections, while the disseminated infection comprises the skin, lungs and other viscera and the larynx ${ }^{12,13}$.

Our patient had a 32-year history of a large verrucous plaque remarkably similar to chromomycosis. Lillis et al. ${ }^{14}$ reported a 29-year-old male South Pacific Islander with an 18-year history of a similar lesion on the left leg, simulating psoriasis, chromomycosis or tuberculosis verrucose cutis. Lee and Brennan ${ }^{15}$ published some cases of otherwise healthy South Pacific Islanders with chronic lesions (more than 20 years) of verrucous plaques on the lower extremities.

In France, 63 cases of culture-confirmed M. marinum infections were studied from January 1, 1996, to December 31,1998 . Nodules were observed in $41(67 \%)$ of the 63 patients, including a sporotrichoid aspect in $16(39 \%$ of the nodules). Other skin lesions were ulcers, abscesses and pustules. Adenitis was observed in 10 patients ${ }^{16}$. It is noteworthy that the skin lesions caused by $M$. marinus in our case were not reported in the other case studies, demonstrating that this characteristic is rare.

Skin lesions of non-tuberculous mycobacteria often go misdiagnosed. Mycobacterium marinum infection is a diagnosis that must be considered as a differential diagnosis with wart tuberculosis, sporotrichosis, chromomycosis, squamous cell carcinoma, leishmaniasis, tularemia, sarcoidosis and reactions to foreign bodies, as the growth of mycobacterium marinus is slow, delaying the diagnosis and treatment ${ }^{17}$. As our case shows, the same immunocompetent patient suffered 32 years with the infection and had a considerable delay in the diagnosis.

A delay between the onset of injuries and the patient's seeking medical attention is due to the fact that the infection is indolent, and the lesions are painless. The diagnosis of $\mathrm{M}$. marinum infection is often delayed, due to its low prevalence and because there are few specific clinical signs and symptoms. Thus, many patients initially receive incorrect diagnoses and inappropriate treatments ${ }^{18}$. Biopsies for tissue cultures and histopathologic examination are essential for the diagnosis 8 . Common histopathological patterns include granulomatous inflammatory infiltrates. Microscopic examination of the specimen after Ziehl-Neelsen staining is positive in $30 \%$ of the cases. A definitive diagnosis is obtained by the finding of a positive culture that occurs in $70-80 \%$ of the cases. After isolation of a photochromogenic Mycobacterium, molecular identification using DNA analysis is necessary ${ }^{12}$. In our case, the REA method was used. PCR amplification and restriction enzyme analysis (PCR-REA), hybridization with species-specific oligonucleotide probes (with or without prior DNA amplification) and nucleic acid sequencing have shown to identify a limited number of Mycobacterium strains ${ }^{19}$.

Mycobacterium marinum isolates are susceptible to rifampin, rifabutin and ethambutol; intermediately susceptible to streptomycin; and resistant to isoniazid and pyrazinamide. Isolates are also susceptible to clarithromycin, sulfonamides or trimethoprim sulfamethoxazole, and susceptible or intermediately susceptible to doxycycline and minocycline. A reasonable approach is a treatment regimen composed of two active agents for 1 to 2 months after resolution of symptoms, typically 3 to 4 months. In a French study, the combination of clarithromycin and rifampin for an average of 3.5 months resolved infections in $93 \%$ of the patients with localized forms and in $72 \%$ of those with deep structure involvement (e.g., osteomyelitis). Treatment failure was related to deep structure involvement, but not to any antibiotic regimen. Clarithromycin and ethambutol are likely to provide the optimal balance of efficacy and tolerability for most patients, with the addition of rifampin in cases of osteomyelitis or other deep structure infection ${ }^{20}$.

Experience in treatment of other NTM suggests that azithromycin may be a reasonable alternative to clarithromycin. Susceptibility tests are not routinely recommended and should be reserved for cases of treatment failure. Resection and debridement of the lesion are generally not recommended and are only indicated in cases refractory to treatment with antibiotics. Some studies showed worsening of conditions when resections and debridement were carried out. Evidently, the intervention may be indicated as an adjunctive treatment in cases of tissue necrosis and septic arthritis, facilitating the effects of antibiotics ${ }^{20}$. Cryotherapy, laser and photodynamic therapy have been reported as effective treatment alternatives, but there are few studies evaluating the efficiency of these methods ${ }^{6}$.

\section{CONCLUSION}

Mycobacterium marinum is considered an uncommon cause of skin infection in humans. The diagnosis requires a high index of suspicion. This infection should be included in the differential diagnosis of chronic verrucous plaques on the extremities, especially if there is a history of exposure 
to fresh, salt water or marine animals. Further studies are needed to understand its clinical polymorphism with some patients showing spontaneous cure, while others develop long-term diseases.

\section{ACKNOWLEDGMENTS}

We would like to thank the Hospital Giselda Trigueiro and Hospital Universitário Onofre Lopes for their technical assistance.

\section{FUNDING}

This case report received no specific grant from any funding agency in the public, commercialor not-for-profit sectors.

\section{CONFLICT OF INTERESTS}

The authors declare that they have no conflict of interests.

\section{REFERENCES}

1. Sander M, Isaac-Renton JL, Sander MA. Atypical clinical and laboratory features of fish-tank granuloma: a case report. SAGE Open Med Case Rep. 2018;6:2050313X1880407.

2. Yoshida M, Fukano H, Miyamoto Y, Shibayama K, Suzuki M, Hoshino Y. Complete genome sequence of Mycobacterium marinum ATCC 927T, obtained using Nanopore and Illumina sequencing technologies. Genome Announc. 2018;6:e0039718.

3. Sette CS, Wachholz PA, Masuda PY, Figueira RB, Mattar FR, Ura DG. Mycobacterium marinum infection: a case report. J Venom Anim Toxins Incl Trop Dis. 2015;21:7.

4. Jaled MM, Pedrini Cinqualbrez MF, González P, Förster Fernández J, Anaya JS, Stengel FM. Infección por Mycobacterium marinum: características epidemiológicas, clínicas y tratamiento. Med Cutan Iber Lat Am. 2010;38:70-5.

5. Das S, Pettersson BM, Behra PR, Mallick A, Cheramie M, Ramesh $\mathrm{M}$, et al. Extensive genomic diversity among Mycobacterium marinum strains revealed by whole genome sequencing. Sci Rep. 2018;8:12040.

6. García Acebes CR, Barchino Ortiz L, Aboín González S, Díaz Ley B, Ruiz Fernández P, Sánchez de Paz F. Infección por Mycobacterium marinum: presentación de un nuevo caso y revisión de la literatura. Actas Dermosifiliogr. 2006;97:653-7.
7. Slany M, Jezek P, Bodnarova M. Fish tank granuloma caused by mycobacterium marinum in two aquarists: two case reports. Biomed Res Int. 2013;2013:161329.

8. Chira-Romero F, Silva CJ, Salinas-Cevallos J. Mycobacterium marinum: reporte de un caso. Rev Argent Dermatol. 2018;99:110.

9. Rallis E, Koumantaki-Mathioudaki E: Treatment of Mycobacterium marinum cutaneous infections. Expert Opin Pharmacother. 2007;8:2965-78.

10. Conforti C, Zalaudek I, Vichi S, Di Meo N. Dermoscopy of Mycobacterium marinum skin infection: a challenging diagnosis. Acta Dermatovenerol Croat. 2019;27:278-9.

11. Aubry A, Mougari F, Reibel F, Cambau E. Mycobacterium marinum. Microbiol Spectr. 2017;5.

12. Holden IK, Kehrer M, Andersen AB, Wejse C, Svensson E, Johansen IS. Mycobacterium marinum infections in Denmark from 2004 to 2017: a retrospective study of incidence, patient characteristics, treatment regimens and outcome. Sci Rep. 2018;8:6738.

13. Hashish E, Merwad A, Elgaml S, Amer A, Kamal H, Elsadek A, et al. Mycobacterium marinum infection in fish and man: epidemiology, pathophysiology and management; a review. Vet Q. 2018;38:35-46.

14. Lillis JV, Winthrop KL, White CR, Simpson EL. Mycobacterium marinum presenting as large verrucous plaques on the lower extremity of a South Pacific Islander. Am J Trop Med Hyg. 2008;79:166-7.

15. Lee MW, Brenan J. Mycobacterium marinum: chronic and extensive infections of the lower limbs in South Pacific Islanders. Australas J Dermatol. 1998;39:173-6.

16. Aubry A, Chosidow O, Caumes E, Robert J, Cambau E. Sixtythree cases of Mycobacterium marinum infection: clinical features, treatment, and antibiotic susceptibility of causative isolates. Arch Intern Med. 2002;162:1746-52.

17. Lesmes BL, Ramírez AF, Rolon M, Castro E. Mycobacterium marinum y lesión verrugosa simulando carcinoma escamocelular. Rev Colomb Cancerol. 2004;8:45-51.

18. Veraldi S, Pontini P, Nazzaro G. Amputation of a finger in a patient with multidrug-resistant Mycobacterium marinum skin infection. Infect Drug Resist. 2018;11:2069-71.

19. Sayin Z, Erganis O. Identification of Mycobacterium strains by PCR and PCR-REA. Bull Vet Inst Pulawy. 2011;55:641-4.

20. Griffith DE, Aksamit T, Brown-Elliott BA, Catanzaro A, Daley C, Gordin F, et al. An official ATS/IDSA statement: diagnosis, treatment, and prevention of nontuberculous mycobacterial. Am J Respir Crit Care Med. 2007;175:367-416. 